P549

\section{Intra-Arterial Thrombolysis for the Treatment of Frostbite Injuries}

\section{Sarah M. Khoncarly, Issac Dahan \\ MetroHealth Medical Center, Case Western Reserve University, Cleveland, United States. \\ E-mail: sarahkhoncarly@gmail.com}

Educational Poster Background: Frostbite is a spectrum of localized cold thermal injuries as a result of severe cold exposure and tissue freezing. These injuries can be challenging for clinicians to treat as they can vary in clinical presentation. Clinical examination alone is not helpful in determining the extent of injury, and diagnostic imaging, particularly angiography, is extremely helpful in guiding management and delineating treatment. If not identified and treated early, the effects of frostbite are detrimental and can have significant negative impacts on limb functionality and the quality of life of those effected. Historically, the poor prognosis of this phenomenon leads to inevitable surgical amputation as traditional treatments are unable to mitigate the perpetual tissue ischemia and eventual tissue loss. Intra-arterial tissue plasminogen (tPA) thrombolysis has recently been employed by interventional radiology as a successful treatment option for frostbite as it prevents the formation of microvascular thrombosis and maintains adequate blood flow to the peripheral structures. Here, we present example cases of intra-arterial tPA thrombolysis as an effective treatment for frostbite and outline the clinical presentations, diagnostic imaging, and posttreatment outcomes. Consultation with interventional radiology, especially at the initial clinical evaluation, can aid clinicians in decisionmaking and guide appropriate imaging and treatments to better delineate effective and efficient disease management and hopefully delay or even prevent surgical amputation and permanent injury.

1. To provide an overview of the presentation, pathophysiology, and diagnostic imaging features of frostbite injuries

2. To describe the role interventional radiology plays in the treatment and management of frostbite injuries

3. To outline the indications, procedure technique, and potential complications of intra-arterial thrombolysis and review case examples.
P550

Primary Orbital Atherectomy for Difficult
Chronic Total Occlusion Crossing

Thomas Geisbush, Bulent Arslan1, Ulku C. Turba $^{1}$, Jordan C. Tasse ${ }^{1}$, Sreekumar Madassery ${ }^{1}$

Rush University Medical Center, Chicago, IL, 'IKaweah Delta Health

Care District, Visalia, CA, United States.

E-mail: sreekumar_madassery@rush.edu

Educational Poster Background: With an increasing volume of lower extremity critical limb ischemia (CLI) revascularizations performed, new techniques are required for heavily calcified chronic total occlusions (CTO). We intend to investigate the feasibility of initial orbital atherectomy over low-profile guidewires, despite intraluminal or subintimal tracts, during CTO revascularizations that are resistant to support catheters/balloon advancement in CLI patients. This has been experienced even with retrograde flossing wire access. With the Institutional Review Board approval, angiograms from 2013 to 2018 were reviewed. Patients with CLI $(n=16$, mean age $=69.2$, standard deviation age $=12.7,75.0 \%$ male [12/16], 25.0\% female [4/16]) were identified. Rutherford classification of the patients included 1 Category $4(6.3 \%), 8$ Category $5(50.0 \%)$, and 7 Category $6(37.5 \%) .17$ occlusions were identified in the following arteries ( 2 in one patient) including eight anterior tibial (50.0\%), four posterior tibial (25.0\%), two popliteal $(12.5 \%)$, two peroneal (12.5\%), and one tibioperoneal trunk $(6.7 \%)$. Subintimal course was noted in six cases $(37.5 \%)$. Following lowprofile guidewire crossing, failure of support or balloon catheter advancement was experienced, including coronary balloon catheters. Orbital atherectomy was then performed with a 1.25 or $1.50 \mathrm{~mm}$ Diamondback orbital atherectomy device (Cardiovascular Systems Inc., St. Paul, MN, USA), or with the addition of TruePath (Boston Scientific, Boston, MA, USA) in one case, followed by angioplasty and stenting as warranted. Final angiograms demonstrated successful revascularization of the desired vessel in 16 of the 17 procedures (94\%). Recanalization was not attained in one anterior tibial artery comprised three distinct occlusions. There were no complications. This retrospective analysis provides preliminary evidence of primary orbital atherectomy feasibility over low-profile guidewire CTO crossing, even if partially subintimal. This technique provides the dual benefit of pretreating the vessel before balloon angioplasty and/ or stenting, as well as facilitating device exchange. 